\title{
EMPHASIS ON ANTIBIOTIC OPTIMISATION IN DIFFICULT TO TREAT METHICILLIN RESISTANT STAPHYLOCOCCUS AUREUS INFECTION
}

\author{
Surinder Kumar1, Diksha Budhani², Pallavi Sayal ${ }^{3}$, Pooja Sindwani ${ }^{4}$, Piyush Kumar Jain ${ }^{5}$
}

${ }^{1} H O D$, Department of Microbiology, BPS GMC for Women, Khanpur Kalan, Sonepat, Haryana.

${ }^{2}$ Demonstrator, Department of Microbiology, BPS GMC for Women, Khanpur Kalan, Sonepat, Haryana.

${ }^{3}$ Demonstrator, Department of Microbiology, BPS GMC for Women, Khanpur Kalan, Sonepat, Haryana.

${ }^{4}$ Demonstrator, Department of Forensic Medicine, BPS GMC for Women, Khanpur Kalan, Sonepat, Haryana.

${ }^{5}$ Demonstrator, Department of Pathology, BPS GMC for Women, Khanpur Kalan, Sonepat, Haryana.

ABSTRACT
BACKGROUND
Emergence of serious infections caused by Gram-positive pathogens is increasing despite remarkable advances in antimicrobial
agents. Of great concern is increasing incidence of Community acquired Methicillin Resistant Staphylococcus aureus (MRSA)
infection, due to its growing frequency and its poor associated outcome.
This study aims to analyse resistance pattern of Staphylococcus aureus (S. aureus) isolates in an attempt to understand
prevalence of MRSA from tertiary care centre.
The objective of this study is to measure the severity, antimicrobial susceptibility of S. aureus isolates from a tertiary care
centre and to discuss preventive measure to control its transmission.

\section{MATERIALS AND METHODS}

Present study was carried out in the Department of Microbiology (Study duration- 1 yr., Jan 2015 - Dec 2015), total of 252 nonduplicate S. aureus isolates from various clinical specimens (pus, blood, sterile site fluids) were included in the study. Isolates were identified by standard microbiological techniques. All S. aureus isolates were subsequently tested for methicillin resistance by cefoxitin $(30 \mu \mathrm{g})$ discs test on Mueller-Hinton agar (MHA). Zone diameter of $\leq 21 \mathrm{~mm}$ was reported as MRSA and $\geq 22 \mathrm{~mm}$ was considered as sensitive as per Clinical and Laboratory Standards Institute (CLSI). All isolates were further screened for susceptibility pattern by Kirby-Bauer disc diffusion method on MHA. The antibiotics used were ampicillin (AMP)10 $\mu$ g; linezolid (Lz) $30 \mu \mathrm{g}$; vancomycin (V) $30 \mu \mathrm{g}$; ciprofloxacin (Cf) $5 \mu \mathrm{g}$; gentamicin (G) $30 \mu \mathrm{g}$; Azithromycin (Az) $15 \mu \mathrm{g}$; and Erythromycin (E) 15 $\mu \mathrm{g}$ (HiMedia, India). S. aureus ATCC 25923 was used as control strain and results were interpreted according to CLSI.

\section{RESULTS}

Among MRSA isolates 70/118 (59.32\%), 48/118 (40.67\%), 46/118 (38.98) and 58/118 (49.15\%) were resistant to $\beta$-lactam, aminoglycosides, fluoroquinolones and macrolides respectively. However, lower resistance was seen in oxazolidinones 5 (4.23\%) and all isolates were susceptible to glycopeptide (Vancomycin).

\section{CONCLUSION}

MRSA continues to be a growing burden for the health-care system due to the poor prognosis and high costs associated with this infection. Higher resistance to aminoglycosides, fluoroquinolones and macrolides could be due to selective antibiotic pressure. To our relief, none of our isolates were pandrug resistant.

\section{KEYWORDS}

MRSA, S. Aureus, Pandrug Resistance, Cefoxitin.

HOW TO CITE THIS ARTICLE: Kumar S, Budhani D, Sayal P, et al. Emphasis on antibiotic optimisation in difficult to treat methicillin resistant staphylococcus aureus infection. J. Evolution Med. Dent. Sci. 2017;6(71):5055-5058, DOI: 10.14260/Jemds/2017/1099

\section{BACKGROUND \\ Antimicrobial therapy is the cornerstone for the development of complex clinical interventions in critically ill patients. The discovery and commercialisation of antimicrobials has revolutionised modern medicine, whereas microbial resistance towards them has emerged as an evolutionary response.}

Financial or Other, Competing Interest: None.

Submission 19-06-2017, Peer Review 22-08-2017,

Acceptance 28-08-2017, Published 04-09-2017.

Corresponding Author:

Diksha Budhani,

Demonstrator, Department of Microbiology,

BPS GMC for Women, Khanpur Kalan,

Sonepat, Haryana.

E-mail: diksha.budhani25@gmail.com

DOI: $10.14260 /$ jemds/2017/1099

\section{(c) (1) $(9)$}

Unfortunately, emergence of drug resistance is now considered as major threat that has jeopardised care of thousands of patients worldwide. ${ }^{1}$ Currently, the most notorious superbug is the Gram-positive organism Staphylococcus aureus (S. aureus). ${ }^{2}$

\section{Emergence of Drug Resistance}

Emergence and Evolution of Resistance among S. aureus isolates can be Categorised as:

- The first wave began in mid-1940s with emergence of penicillin-resistance, because of plasmid encoded penicillinase that hydrolyses the beta-lactam ring of penicillin essential for its antimicrobial activity. Penicillin-resistant strains then were observed to cause community infections, by 1950s and 1960s they had become pandemic. ${ }^{3}$ 
- Introduction of methicillin marks the onset of the second wave of resistance. First reports of methicillin resistance were published in 1961. Methicillin resistance determinants which encodes low affinity penicillin binding protein, PBP2A by mecA gene was not identified until more than 20 years later. Unlike penicillinasemediated resistance, which is narrow in its spectrum, methicillin resistance is broad beta-lactam antibiotic class resistance to penicillins, cephalosporins and carbapenems. ${ }^{3}$

\section{Origin of mecA Gene}

Phylogenetic distribution of the mecA homologues suggests that mecA had been vertically transmitted as an ortholog for some time during the course of speciation of sciuri group staphylococcal species such as Staphylococcus fleurettii, Staphylococcus vitulinus, S. sciuri subspecies sciuri and Staphylococcus carnaticus. As the vertically transmitted ortholog, mecA, mecA1, and mecA2 are located at the corresponding loci on the chromosomes of the sciuri group species; S. fleurettii, S. sciuri and S. vitulinus respectively. They have $99.8 \%, 80 \%$ and $91 \%$ nucleotide identities, respectively, to the mecA gene carried by SCCmec on the MRSA chromosome. Thus, apparently S. fleurettii mecA was the original mecA, which was adopted as the methicillinresistance determinant of the SCCmec that converted S. aureus into MRSA. ${ }^{4}$

\section{Evolution of Staphylococcus Species and Loss of mecA Gene}

Staphylococcal species exhibit evident host-specific colonisation. In ongoing ecological study of staphylococci in mammalian species, the appearance of genus Staphylococcus was estimated to be about 250 million years ago by molecular clock method using genome-wide datasets. Staphylococcal species seem to have started to colonise and co-evolve with mammals that emerged almost simultaneously about 225 million years ago. ${ }^{5}$

It is probable that the antecedents of staphylococci e.g. macrococcal species and old staphylococcal species of $\mathrm{S}$. sciuri group required the benefit of mecA or mecC genes to protect themselves from $\beta$-Lactam-producing environmental microorganisms before their descendants successfully adapted to mammalian hosts. The descendant staphylococcal species after successful adaptation as mammalian microbial flora, lost mecA or mecC gene, because they became protected from the assault of $\beta$-lactam producing microorganisms. However, situation changed in the 1940s, when humans started to use penicillin G, threatening colonising staphylococci. They first acquired penicillinase plasmid. Since then the introduction of methicillin in 1960, S. aureus had to regain mecA gene from $S$. fleurettii via the SCCmec. ${ }^{5}$

MRSA is not restricted to any geographical location, but distributed worldwide and now considered endemic to most hospitals (Hospital acquired MRSA). As time progressed, S. aureus not only evolved resistance to number of antibiotic agents, but also transcended to produce infection in healthy members of community (Community acquired MRSA). ${ }^{6}$

Most striking situation is that MRSA strains have emerged with concomitant resistance to many commonly used antibiotics of groups, aminoglycosides, macrolides, fluoroquinolones, chloramphenicol and tetracycline. A special rule has been applied in defining antimicrobial resistance in $\mathrm{S}$. aureus. Once a S. aureus isolate is characterised as an MRSA, it is instantly classified as multidrug resistant (MDR), because resistance to oxacillin or cefoxitin infers nonsusceptibility to all categories of $\beta$-lactam antimicrobials Thus, MDR-MRSA is the new or rather a continually evolving paradigmatic pathogen. ${ }^{7}$ An utterly sad fact today is that more than 95\% MRSA worldwide do not respond to the firstline antibiotics. ${ }^{8}$

\section{Objective}

To measure the severity, antimicrobial susceptibility of $S$. aureus isolates from a tertiary care centre and to discuss preventive measure to control its transmission.

\section{MATERIALS AND METHODS \\ Study Design}

This descriptive study was carried out from Jan 2015 - Dec 2015 in a tertiary care centre. Study group includes patients of all age groups attending Inpatient and Outpatient department.

\section{Sample Details}

A total of 4067 non-duplicate clinical specimens (pus, blood, body fluid specimens) sent to the Microbiology Laboratory over the study period were processed by conventional microbiological techniques. ${ }^{9}$ Urinary S. aureus isolates were not included in our study as- (1) There was no patient of symptomatic urinary tract infection concurrent with isolation of S. aureus, (2) Staphylococcal bacteraemia concomitant with isolation of S. aureus from urine was not established.

\section{Isolation and Identification}

The samples were inoculated onto blood agar and MacConkey's agar (HiMedia, India). Plates were incubated at $37^{\circ} \mathrm{C}$ for $18-24$ hours. S. aureus was identified and differentiated from related organisms on the basis of colony morphology, Gram staining, catalase test, slide and tube coagulase test and mannitol fermentation. ${ }^{9}$ S. aureus ATCC 25923 was used as control strain.

\section{Antibiotic Susceptibility Test by Kirby-Bauer Method}

The isolates were subjected to susceptibility testing by KirbyBauer disc diffusion method on Mueller-Hinton agar plates and the results were interpreted according to the guidelines of the Clinical Laboratory Standard International (CLSI). ${ }^{10}$ The antibiotics used were ampicillin (AMP) $10 \mu \mathrm{g}$; linezolid (Lz) $30 \mu \mathrm{g}$; vancomycin (V) $30 \mu \mathrm{g}$; ciprofloxacin (Cf) $5 \mu \mathrm{g}$; gentamicin (G) $30 \mu \mathrm{g}$; Azithromycin (Az) $15 \mu \mathrm{g}$; and Erythromycin (E) $15 \mu \mathrm{g}$ (HiMedia, India). S. aureus ATCC 25923 was used as control strain.

\section{Detection of Methicillin Resistance by Phenotypic Method}

All the S. aureus isolates were subsequently tested for methicillin resistance by cefoxitin $(30 \mu \mathrm{g})$ discs.

\section{Cefoxitin Disc Test}

All the isolates were subjected to cefoxitin disc diffusion test using a $30 \mu \mathrm{g}$ disc. A $0.5 \mathrm{McF}$ arland standard suspension of the isolate was made and lawn culture was done on MHA plate. Plate was incubated at $37^{\circ} \mathrm{C}$ for 18 hours and zone diameter was measured. An inhibition zone diameter of $\leq 21$ 
mm was reported as Cefoxitin resistant and $\geq 22 \mathrm{~mm}$ was considered as sensitive. ${ }^{10}$

\section{Statistical Analysis}

Statistical package for social sciences (SPSS) version 18 was used to analyse our data. Clinical outcomes were compared statistically using Chi-square test. $\mathrm{P}<0.05$ was taken as "statistically significant."

\section{RESULTS}

Out of 4067 samples processed, 252 S. aureus strains were isolated. Isolation rate of S. aureus was maximum in blood $(41.79 \%)$ followed by pus $(31.13 \%)$. Details of sample distribution are mentioned in Table 1. Isolation rate of MRSA was different among various samples. Maximum was found in pus $(55.29 \%)$ followed by blood $(30.37 \%)$ samples.

\begin{tabular}{|c|c|c|c|c|}
\hline Specimen & $\begin{array}{c}\text { Total } \\
\text { Samples }\end{array}$ & $\begin{array}{c}\text { Culture } \\
\text { Positive }\end{array}$ & $\begin{array}{c}\text { Staphylo- } \\
\text { coccus } \\
\text { aureus }\end{array}$ & MRSA \\
\hline Pus & 1687 & $\begin{array}{c}546 \\
(32.36 \%)\end{array}$ & $\begin{array}{c}170 \\
(31.13 \%)\end{array}$ & $\begin{array}{c}94 \\
(55.29 \%)\end{array}$ \\
\hline $\begin{array}{c}\text { Body fluid } \\
\text { (Pleural, } \\
\text { Ascitic, } \\
\text { Synovial) }\end{array}$ & 493 & $\begin{array}{c}45 \\
(9.72 \%)\end{array}$ & $\begin{array}{c}3 \\
(6.66 \%)\end{array}$ & Nil \\
\hline Blood & 1887 & $\begin{array}{c}189 \\
(10.01 \%)\end{array}$ & $\begin{array}{c}79 \\
(41.79 \%)\end{array}$ & $\begin{array}{c}24 \\
(30.37 \%)\end{array}$ \\
\hline \multicolumn{5}{|c|}{ Table 1. Sample Distribution } \\
\hline
\end{tabular}

Age wise distribution of patients from whom different MRSA/MSSA isolates were made is depicted in Table 2.

\begin{tabular}{|c|c|c|c|}
\hline $\begin{array}{c}\text { Age } \\
\text { (in years) }\end{array}$ & $\begin{array}{c}\text { MRSA Isolates } \\
\quad(n=118)\end{array}$ & $\begin{array}{c}\text { MSSA Isolates } \\
\quad(n=134)\end{array}$ & P-value \\
\hline$<15$ & $27(22.88 \%)$ & $55(41.04 \%)$ & \multirow{5}{*}{0.022} \\
\hline $16-30$ & $44(37.28 \%)$ & $42(31.34 \%)$ & \\
\hline $31-45$ & $20(16.94 \%)$ & $16(11.94 \%)$ & \\
\hline $46-60$ & $19(16.10 \%)$ & $11(8.20 \%)$ & \\
\hline $61-75$ & $8(6.77 \%)$ & $10(7.46 \%)$ & \\
\hline
\end{tabular}

Table 2. Age Wise distribution of Staphylococcal Isolates

\begin{tabular}{|c|c|c|c|}
\hline Gender & MRSA & MSSA & P-value \\
\hline Male (n=137) & $63(45.98 \%)$ & $69(50.36 \%)$ & 0.763 \\
\hline $\begin{array}{c}\text { Female } \\
(\mathrm{n}=115)\end{array}$ & $55(47.82 \%)$ & $65(56.52 \%)$ & 0.763 \\
\hline \multicolumn{3}{|c|}{ Table 3. Gender Wise distribution of Staphylococcal } \\
Isolates \\
\hline
\end{tabular}

In our study, $45.98 \%$ MRSA isolates were from males and $47.82 \%$ isolates were recovered from females. However, no statistical significant difference was observed among males and females. Our study showed highest percentage (37.28\%) of MRSA occurrence in patients with age group $16-30$ years [Table 3].

\begin{tabular}{|c|c|c|c|c|c|}
\hline Antibiotic Category & \multicolumn{2}{|c|}{ Antimicrobial Agents } & $\begin{array}{c}\text { MRSA } \\
(n=118)\end{array}$ & $\begin{array}{c}\text { MSSA } \\
(n=134)\end{array}$ & P-value \\
\hline \multirow{2}{*}{$\beta$-lactam } & \multirow{2}{*}{ Ampicillin } & $\mathrm{S}$ & $48(40.67 \%)$ & $60(44.77 \%)$ & \multirow{2}{*}{0.511} \\
\hline & & $\mathrm{R}$ & $70(59.32 \%)$ & $74(55.22 \%)$ & \\
\hline \multirow{2}{*}{ Fluoroquinolone } & \multirow{2}{*}{ Ciprofloxacin } & $\mathrm{S}$ & $70(59.32 \%)$ & $73(54.47 \%)$ & \multirow{2}{*}{0.438} \\
\hline & & $\mathrm{R}$ & $48(40.67 \%)$ & $61(45.52 \%)$ & \\
\hline \multirow{2}{*}{ Aminoglycoside } & \multirow{2}{*}{ Gentamicin } & $\mathrm{S}$ & $72(61.01 \%)$ & $79(58.95 \%)$ & \multirow{2}{*}{0.738} \\
\hline & & $\mathrm{R}$ & $46(38.98 \%)$ & $55(41.04 \%)$ & \\
\hline \multirow{4}{*}{ Macrolide } & \multirow{2}{*}{ Erythromycin } & $\mathrm{S}$ & $60(50.84 \%)$ & $89(66.41 \%)$ & \multirow{2}{*}{0.012} \\
\hline & & $\mathrm{R}$ & $58(49.15 \%)$ & $45(33.58 \%)$ & \\
\hline & \multirow{2}{*}{ Azithromycin } & $\mathrm{S}$ & $63(53.38 \%)$ & $87(64.92 \%)$ & \multirow{2}{*}{0.062} \\
\hline & & $\mathrm{R}$ & $55(46.61 \%)$ & $47(35.07 \%)$ & \\
\hline \multirow{2}{*}{ Oxazolidone } & \multirow{2}{*}{ Linezolid } & $\mathrm{S}$ & $113(95.76 \%)$ & $131(97.76 \%)$ & \multirow{2}{*}{0.366} \\
\hline & & $\mathrm{R}$ & $05(4.23 \%)$ & $03(2.23 \%)$ & \\
\hline \multirow{2}{*}{ Glycopeptide } & \multirow{2}{*}{ Vancomycin } & $\mathrm{S}$ & $118(100 \%)$ & $134(100 \%)$ & \multirow{2}{*}{ NA } \\
\hline & & $\mathrm{R}$ & 0 & 0 & \\
\hline \multicolumn{6}{|c|}{ robial susceptibility Pattern of S. aureus Isolates } \\
\hline
\end{tabular}

S-susceptible, R-resistant

Resistance pattern of MRSA isolates showed that 70/118 (59.32\%), 48/118 (40.67\%), 46/118 (38.98), 58/118 $(49.15 \%)$ were resistant to $\beta$-lactam, aminoglycosides, fluoroquinolones and macrolides respectively. However, lower resistance was seen in oxazolidinones 5 (4.23\%) and all isolates were susceptible to glycopeptide (Vancomycin). Also, we observed coexisting resistance to different antibiotics with cefoxitin was higher in methicillin resistant isolates compared to methicillin sensitive isolates [Table 4].

\section{DISCUSSION}

Out of 252 Staphylococcus aureus isolates, 118 strains were MRSA. S. aureus were mainly isolated from blood sample
(41.79\%) followed by pus sample (31.13\%) [Table 1]. Our findings were consistent with study conducted by Sangeeta Joshi et al who also demonstrated S. aureus was mainly isolated from skin and soft tissue infections (64\% in 2008 and $61 \%$ in 2009) followed by blood. ${ }^{11}$

Distribution of the MRSA was higher among females $(47.8 \%)$ than males $(45.9 \%)$ [Table 3]. But gender wise, no statistical significance was observed. Similar findings were observed by Dardi Charan Kaur et al, which also concluded that MRSA was higher among females $(19,52.78 \%)$ as compared to males $(17,47.22 \%) .^{12}$

Among MRSA isolates (59.32\%), (40.67\%), (38.98\%) and $(49.15 \%)$ strains were also resistant to $\beta$-lactam, aminoglycosides, fluoroquinolones and macrolides respectively. However, lower resistance was seen against 
oxazolidinones (4.23\%), whereas all isolates were susceptible to glycopeptide (Vancomycin) [Table 4]. Dardi Charan Kaur et al also observed that none of their 36 isolates were resistant to antimicrobial category like glycopeptides, glycylcyclines and fucidanes. But $100 \%$ resistance to fluoroquinolones, aminoglycosides and macrolides was noted, while lincosamides showed $97.22 \%$ resistance. Lower resistance was seen in case of oxazolidinones (2.78\%), streptogramins (5.56\%) and lipopeptides (5.56\%). ${ }^{12}$

\section{CONCLUSION}

Our study demonstrated high isolation rate of MRSA with high rate of resistance to commonly used anti-staphylococcal agents. To prevent the emergence of pandrug resistance among isolates, we recommend-

- Although colonisers are generally asymptomatic, they are a potential reservoir of infections. Since MRSA strains spread readily in hospitals from colonised or infected persons, proper screening for MRSA among healthcare workers and patients should be done.

- The selection of antimicrobial agent should be based on in vitro susceptibility and the hospital-based antibiotic policies must be strictly followed and constant surveillance of drug resistance for all bacterial pathogens is needed.

- Large amount of unnecessary antibiotics should not be taken.

- National surveillance of antibiotic resistance and antibiotic use.

- Setting up and strengthening infection control committees in hospitals.

- Antimicrobial stewardship programs can be implemented to reduce inappropriate use of antimicrobials, thereby controlling the development of resistance.

\section{REFERENCES}

[1] Munita JM, Bayer AS, Arias CA. Evolving resistance among gram positive pathogens. CID 2015;61(Suppl 2):S48-57.

[2] Davies J, Davies D. Origin and evolution of antibiotic resistance. Microbiol Mol Biol Rev 2010;74(3):417-33.
[3] Chambers HF, Deleo FR. Waves of resistance: staphylococcus aureus in the antibiotic era. Nat Rev Microbiol 2009;7(9):629-41.

[4] Tsubakishita S, Kuwahara-Arai K, Sasaki T, et al. Origin and molecular evolution of the determinant of methicillin resistance in staphylococci. Antimicrob Agents Chemother 2010;54(10):4352-9.

[5] Hiramatsu $\mathrm{K}$, Hanaki $\mathrm{H}$, Ino $\mathrm{T}$, et al. Methicillin resistant staphylococcus aureus clinical strain with reduced vancomycin susceptiblity. J Antimicrob Chemother 1997;40(1):135-6.

[6] Deurenberg RH, Stobberingh EE. The evolution of staphylococcus aureus. Infect Genet Evol 2008;8(6):747-63.

[7] Debasmita D, Shakti R, Mahesh CS, et al. Surveillance of infection status of drug resistant staphylococcus aureus in an Indian teaching hospital. Asian Pac J Trop Dis 2013;3(2):133-42.

[8] David MZ, Daum RS. Community-associated methicillin resistant staphylococcus aureus: epidemiology and clinical consequences of an emerging epidemic. Clin Microbiol Rev 2010;23(3):616-87.

[9] Collee JG, Duguid JP, Fraser AG, et al. Laboratory strategy in the diagnosis of infective syndrome. In Collee JG, Fraser AG, Marmion BP, (eds). Mackie \& McCartney practical medical microbiology. $14^{\text {th }}$ edn. New Delhi: Elsevier, a division of Reed Elsevier India Pvt. Ltd 2006:53-94.

[10] Wayne PA. Clinical and laboratory standard institute 2006. Performance standards for antimicrobial disc tests. Approved standards. $9^{\text {th }}$ edn. Sixteenth informational supplement M2-M9. 2006:P 26.

[11] Joshi S, Ray P, Manchanda V, et al. Methicillin resistant staphylococcus aureus (MRSA) in India: prevalence \& susceptibility pattern. Indian J Med Res 2013;137(2):363-9.

[12] Kaur DC, Chate SS. Study of antibiotic resistance pattern in methicillin resistant staphylococcus aureus with special reference to newer antibiotic. J Glob Infect Dis 2015;7(2):78-84. 